Revue d'histoire de l'Amérique française

REVUE D.HISTOIRE DE L'AMÉRIQUE FRANÇAISE

\title{
Le Bas-Canada et les imprimés (1809-1810)
}

\section{Jean-Pierre Wallot}

Volume 20, numéro 4, mars 1967

URI : https://id.erudit.org/iderudit/302614ar

DOI : https://doi.org/10.7202/302614ar

Aller au sommaire du numéro

Éditeur(s)

Institut d'histoire de l'Amérique française

ISSN

0035-2357 (imprimé)

1492-1383 (numérique)

Découvrir la revue

Citer cet article

Wallot, J.-P. (1967). Le Bas-Canada et les imprimés (1809-1810). Revue d'histoire de l'Amérique française, 20(4), 556-565. https://doi.org/10.7202/302614ar d'utilisation que vous pouvez consulter en ligne.

https://apropos.erudit.org/fr/usagers/politique-dutilisation/ 


\section{LE BAS-CANADA ET LES IMPRIMÉS (1809-1810)}

L'existence d'imprimés dans une contrée d' "ignorants" frise le paradoxe. Prétendre les analyser et pressentir leur influence, n'est-ce pas de l'impudence ? Et pourtant, dans ce Bas-Canada du début du XIXe siècle, supposément bouché, cadenassé contre le reste du monde et lui-même, les imprimés existent, depuis longtemps d'ailleurs. Ils s'avèrent nombreux et semblent infléchir l'opinion publique. Ils ne font pas que délecter quelques cercles restreints de gourmets intellectuels. Ils véhiculent des idées et des informations qui pénètrent les couches populaires - au moins sur le plan politique. Est-ce que, cédant à la tentation toujours présente des généralisations faciles, des contemporains et des historiens ont exagéré le nombre d'illettrés ? Cela semble certain en plusieurs cas. On "télescope" trop aisément les décennies différentes qui jalonnent la fin du XVIIIe et le début du XIXe siècle jusqu'en 1840. Quoi qu'il en soit, un autre facteur est intervenu. Au cours des nombreuses campagnes électorales, ou en dehors d'elles, les candidats élus et défaits, leurs organisateurs, des professionnels, des curés, des habitants plus instruits s'évertuent à répandre leur évangile politique et social en lisant et commentant les journaux de leur choix à ceux qui ne savent pas lire. Pourquoi donc Craig redoute-t-il la distribution gratuite du Canadien dans les campagnes ${ }^{1}$, sinon à cause de ces réseaux capillaires qui alimentent le peuple des campagnes comme celui des villes.

On aurait tort de concevoir ce mouvement dans un seul sens. En fait, il y a échange. D'une part, les opinions des habitants remontent le circuit, s'étalent dans les journaux ou sont exploités par des politiciens habiles. D'autre part, les

1 Craig à Castlereagh (no 29), Québec, 5 août 1808, Archives publiques du Canada [APC], M.G. 11, Q. 107:306 et ss. 
conflits raciaux, politiques, économiques, sociaux et culturels, se répercutent également dans les imprimés. Selon les débats au fil des années, les brochures convergent sur la ou les questionsvedettes: par exemple sur la crise raciale et politique, dans les années 1809-1810; sur la destruction de la colonie de Selkirk et les problèmes du Nord-Ouest, en 1816, 1817 et 1818 .

Dans une série d'articles ${ }^{2}$, nous analyserons quelques brochures parues à Londres ou dans le Bas-Canada en 1809 et 1810 , au plus fort de la crise sous le gouverneur Craig. Elles éclairent singulièrement non seulement le pays lui-même, son climat, ses ressources, ses habitants, mais encore les conflits aigus qui le crispent: conflits qui surplombent les personnalités en présence. Craig ne dresse pas les partis l'un contre l'autre: Britanniques et Canadiens s'assènent de durs coups depuis longtemps et plus particulièrement dans les journaux, depuis la fin de 1806. Déjà, ils ont esquissé toutes les idées principales qui domineront les querelles pour de nombreuses années. Le gouverneur envenime les frictions lorsqu'il veut y mettre un terme en optant pour la colonisation britannique. La crise s'accélère et les antinomies se cristallisent dans des brochures qui, surtout du côté britannique, peuvent mieux en expliciter certains aspects que de brefs articles de journaux.

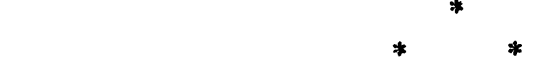

La Société littéraire de Québec convoqua une assemblée pour le 3 juin 1809 afin de célébrer l'anniversaire de naissance du roi George III, "notre Glorieux Souverain" (p. 3). A la fin du mois, elle en édita un compte rendu de 21 pages en très petits caractères ${ }^{3}$. D'abord, Louis Plamondon, secrétaire de la Société,

${ }^{2}$ Chaque article comprendra une analyse d'une brochure ainsi que des citations importantes, dans le texte ou en appendice, selon les cas. Des analyses plus courtes de ces brochures ainsi que de toutes les autres et des journaux imprimés dans le Bas-Canada, de 1801 à 1820, paraîtront dans une étude en préparation sur les Imprimés dans le Bas-Canada, 1801-1820, par John Hare et Jean-Pierre Wallot.

3 Séance de la Société littéraire de Québec, tenue samedi, le se. juin, 1809. Floreamus in Nemoribus. Québec, 1809. Les fautes, dans les citations, sont reproduites du texte original. Nous nous sommes contentés de moderniser l'orthographe. 
burine un panégyrique délirant du roi britannique et de son règne, glorieux tant en matière intérieure que sur la scène internationale: règne "rempli ... de faits que la postérité ne pourra jamais croire" (3). "D'un côté vous verrez constamment un Roi digne de gouverner un semblable peuple, et de l'autre un peuple digne d'être gouverné par un semblable Roi" (4). En 1760 , un peuple anglais heureux, puissant, riche, fut privé subitement de son roi, George II, "couvert de gloire et adoré de ses sujets". Mais le "Dieu tutélaire d'Albion voulait encore son bonheur" (4-5) : George III accéda au trône. Il épousa une princesse "douce, affable, compatissante, et douce de toutes les vertus qui font l'ornement de son sexe" (5). La guerre se clôtura en 1763, grâce aux efforts de paix et aux victoires de l'Angleterre: "Jamais guerre ne fut plus heureuse et ne [lui] fit plus d'honneur..." (5). Elle se retrouvait maîtresse de presque toute l'Amérique.

Après la guerre, George III, "à qui la passion des conquêtes n'a jamais fait verser une seule goutte de sang . . . ne s'occupa plus que du bonheur intérieur de son empire" (6) : progrès prodigieux dans le commerce, les arts, les sciences, l'éducation. Mais voilà que l'Amérique rompit cette "paix profonde" (7): elle "osa se rebeller contre la mère patrie"! Le roi esquissa vainement un rapprochement.

Pour nous, Messieurs, cette époque sera toujours la plus belle de notre histoire, et GEORGE III se souviendra longtemps qu'il trouva dans ses jeunes sujets du Canada, de vieux guerriers qui devinrent les défenseurs de sa nouvelle conquête. Lorsque les Rois eux-mêmes abandonnaient la cause des Rois; Lorsque l'infortuné Louis XVI favorisait imprudemment des rebelles; lorsque l'Espagne envoyait ses flottes à leurs secours, le Canada repoussait vivement ses voisins qui n'étaient plus ses frères. Je sais, Messieurs, que les Canadiens ne firent alors que ce qu'ils devaient faire; mais il semble que nous vivons dans des temps où faire son devoir est une chose digne d'admiration. Il est vrai aussi que le Canada, bien éloigné de sa prospérité actuelle, se trouvait dans une situation très critique, et si l'on pât douter un 
seul instant de son attachement à la Grande-Bretagne, c'était sans doute, dans un temps où venant d'être conquis, sa forme de gouvernement était incertaine et le pouvoir qui le régissait peu connu; dans un temps où des rebelles que [ 8 - ] les succès favorisaient lui offrait l'appât tentatif de la liberté et lui montrait de près un malheureux exemple à suivre. Mais ... si l'Amérique, à qui sa liberté devient déjà à charge, eût joui du bonheur dont nous jouissons; que si la constitution de l'Amérique eût été celle sous laquelle nous vivons, elle n'aurait jamais essayé d'obtenir une meilleure condition $(7-8)$.

Avec la paix de 1783 resurgirent "l'abondance et la prospérité" (8). La tentative ratée d'assassinat du roi, en 1786 , lui révéla "les transports d'amour" (8-9) du peuple à son endroit. George III triompha également de la maladie. Mais voilà qu'un "monstre né pour le malheur du genre humain" - la Révolution française ! - se déchaîna et ravagea la France, puis se déchaîna dans le monde. Le bras protecteur de George III préserva le Canada de ses crimes:

... Un Roi dont tout le crime fut trop de bonté, traîné sur l'échafaud par des sujets avides de son sang; une Reine, le modèle de son sexe, massacrée par les meurtriers de son époux; un jeune Prince l'espoir de la France, empoisonné par les barbares chargés de son éducation; des milliers d'innocentes victimes tombant sous la hâche des bourreaux dans tous les coins de la France; les autels renversés, les tombeaux profanés, l'innocence, la paix, la justice, en un mot toutes les vertus s'envolant de ce malheureux royaume; tel serait le fond d'un tableau que mon pinceau refuserait de tracer... Un seul pays a été respecté par la révolution et cet heureux pays est le nôtre. Dans le temps même que les puissances les plus formidables de l'Europe voyaient leur pouvoir s'échapper de leur main; dans le temps que les Rois les plus puissants chassés de leurs trônes, cherchaient une retraite dans les endroits les plus reculés, GEORGE III étendait son bras bienfaisant 
sur le Canada, et y établissait cette heureuse constitution ${ }^{4}$ qui a été la cause de notre prospérité.

Les peuples des deux hémisphères ont été ravagés par la plus cruelle guerre qui ait jamais existé; les plus puissants empires ont été détruits, les villes les plus florissantes ont été ruinées; la Grande Bretagne même a sacrifié des milliers de ses braves marins et de ses braves soldats; le Canada seul a joui d'un repos que lui a assuré son généreux Souverain. O Meliboee, Deus nobis hoec otia fecit. Oui, Messieurs, [11-] c'est un Dieu qui nous a causé ce bonheur, c'est un Roi que nous ne saurions assez chérir ; c'est un Roi que l'Eternel s'est plu à former pour le bonheur de ses sujets.

... Un homme né pour la terreur du genre humain, a ravagé toutes les nations; il ... a embrasé toutes les parties du globe; ... il a marché à travers les peuples et les a écrasés. La Grande-Bretagne seule s'est moquée de ses projets. Semblable aux vagues qui viennent se briser sur les bords de l'Isle fortunée des Bretons, la haine, la furie de Buonaparte n'a pu atteindre l'intrépide Angleterre...

Il me semble que dans cette dernière guerre, la nature ait voulu former un peuple de héros pour la gloire de la Grande-Bretagne. GEORGE TROIS ... HOWE, DUNCAN ... NELSON ... devant Trafalgar... victoires [qui] annoncent au monde entier que la souveraine des mers fera toujours respecter l'autorité que lui a acquise sa valeur et son industrie.

Et toi brave et malheureuse Espagne ... [12-] adore en silence, le pouvoir de l'Eternel qui veut éprouver ta constance et ta valeur... qui n'admirera la promptitude avec laquelle nous avons vu le peuple Anglais voler au secours des Patriotes Espagnols ... Tel est, Messieurs, le coup d'œil que nous offre la Grande Bretagne depuis le commencement du Règne de GEORGE III. Coup d'œil merveilleux . . . (11-12).

L'orateur claironne enfin un hymne à la "Conquête providentielle", hymne qui, avec ce qui précède, doit être comparé au

4 La constitution de 1791.

Napoléon. 
"sermon" de Mgr Plessis, en 1799, sur la victoire d'Aboukir ${ }^{6}$.

Pour nous, Messieurs, bénissons à jamais l'heureux moment où les armes victorieuses de l'Angleterre, sous l'immortel WOLFE, nous rendirent sujets de l'Empire Britannique. Bénissons à jamais notre heureux sort et reconnaissons que parmi tant de peuples dispersés sur la surface du globe, il n'en ait aucun à qui le maître des Rois ait autant prodigué de bienfaits. Rappellons nous que parmi les heureux sujets même de GEORGE III, il n'en ait aucun qu'il ait comblé de plus de faveurs. La plus belle des constitutions, le plus heureux des repos sont les moindres de ses largesses. Il a voulu que ceux à qui il donnerait le soin de le représenter fussent de ces hommes rares qui savent joindre la douceur à la fermeté, la valeur au savoir et les talents au mérite. Admirons dans SIR JAMES ${ }^{7}$ toutes ces qualités réunies et portons des regards reconnaissants vers la source d'où découlent tant de bienfaits.

N'oublions pas surtout, que lorsque celui qui tient dans ses mains la destinée des peuples, veut punir des nations ingrates, il leur envoie des maîtres qui appesantissent sur elles leur sceptre de fer. Ne méritons [13-] jamais un semblable malheur et méritons au contraire d'être gouvernés longtemps par un Roi, tel que GEORGE III ...

B On trouvera de larges extraits de ce sermon et des commentaires fort pertinents du chanoine Lionel Groulx, dans Notre Maître le passé (Troisième série) (Montréal, 1944) : 125-164.

7 Sir James Craig, gouverneur en chef des Canadas de 1807 à 1811.

8 Les deux derniers paragraphes du discours contiennent des allusions à peine voilées au malaise politique qui oppose de plus en plus le parti canadien à Craig, à l'exécutif, aux députés britanniques et aux quelques Canadiens "à place". Plamondon exalte Craig et sa conduite, donc censure implicitement celle du parti canadien. De plus, il insinue que "l'ingratitude", v.g. les plaintes qui s'exhalent par exemple dans Le Canadien, pourrait entraîner la perte des nombreux privilèges dont jouit, selon lui, le peuple canadien. On ne peut donc que s'étonner des commentaires virulents du Quebec Mercury, l'organe du parti britannique, à l'égard de la Société littéraire. Ce serait une société "politique", "useless if not prejudicial", créée par des gens sans emploi pour soutirer de l'argent: en effet, les membres devaient se cotiser pour ouvrir une salle de lecture (The Quebec Mercury, 10 juillet 1809). L'auteur rageait peut-être parce qu'une telle société ne pouvait qu'encourager l'étude du Français et se débondait d'une loyauté trop bavarde, donc contraire à l'argumentation britannique - que nous cernerons mieux dans des analyses subséquentes de brochures - en 
Après un coup d'encensoir à "une constitution aussi sage", François Romain, président de la Société littéraire, définit le but de la Société: "l'étude de la Littérature" (13). Or, aucun sujet littéraire n'est plus approprié que "celui de célébrer la naissance du plus grand et du plus doux des monarques, de rendre grâce au ciel de l'avoir fait naître, et de le prier de prolonger notre félicité en prolongeant ses jours" (13). Le président décerne ensuite les prix du concours littéraire: 1er prix de poésie anglaise à John Fleming ${ }^{9}$ pour son ode "On the Birth Day of His Majesty (14) ; ler prix de poésie française à Canadiensis ${ }^{10}$ pour son ode sur le même sujet.

Le "poème" de Fleming, lourd et pédant, submerge le roi et Craig de compliments :

... So GEORGE's fost'ring and paternal hand

Dispenses blessings o'er this happy land:

See! Freedom lift her beauteous crest;

See! High enthron'd firm Justice plac'd;

See! Industry and Plenty rise

And wealth and Commerce greet our eyes

And Science, teaching all these gifts to prize;

While like an Angel sent by Heav'n's command,

The brave SIR HENRY guards the happy land...

But not alone for Britain's millions born;

Exalted high on her Imperial Throne,

Let thy strong Arm still Europe's fate controul;

Let thy loud Thunders 'gainst her Tyrants roll

And Justice' voice be heard, from Pole to Pole.

Hail joyful Morn, ordain'd for social mirth,

faveur de l'assimilation des Canadiens: danger que représentent les Canadiens, parce que Français; avantages de l'anglicisation pour l'empire et pour eux-mêmes. Le président de la Société, d'autre part, était portier de la Chambre d'Assemblée.

9 John Fleming (1786-1832), un marchand écossais, émigra au Canada vers 1803 . Il géra la maison Hart, Logan \& Co. et devint président de la Banque de Montréal. Captivé également par les lettres, il rassembla l'une des bibliothèques les mieux pourvues de son époque et rédigea une histoire du Canada inspirée par les intérêts des marchands britanniques, The Political Annals of Lower Canada (Montréal, 1828). Cf. Wallace, The Macmillan Dictionary of Canadian Biography... (3e éd., Toronto, 1963) [à l'avenir: Macmillan Dictionary....], 235.

10 Le président déclara qu'on remettrait la médaille à Canadiensis dès que celui-ci daignerait se faire connaître. Plusieurs articles parurent dans te Canadien, entre 1806 et 1810 , sous ce pseudonyme. 
Auspicious morn, that gave our Sov'reign birth!

The Muse of Canada thee humbly hails;

Thy praise resounds thro' her sweet smiling vales.

L' "Ode" de Canadiensis vaut bien celui de Fleming ! L'auteur contraste l'Europe ensanglantée et le Canada, paisible et prospère sous un roi qui règne par l'amour:

... Ici tout vit en paix sous le meilleur des Rois:

La loyauté m'inspire,

Et ma rustique Lyre

Va par ses premiers sons célébrer GEORGE TROIS.

... D'un Souverain chéri pour honorer la Fête

Que les vœux et la joie éclatent tour-à-tour;

Cet éloge sans feinte

Ne doit rien à la crainte

Citoyen sur le Throne il règne par l'amour.

D'abord le préjugé qu'inspira sa puissance

Fit redouter ses lois à nos pères surpris,

Enfin de sa bonté la douce expérience

Nous apprit d'un tel Prince à sentir tout le prix;

Et d'un peuple fidele

Devenus le modèle

Nous avons combattu, vaincu ses ennemis.

Sur ces fertiles bords oubliés par Bellone

Coulent du St. Laurent les flots majestueux,

Le commerce fleurit et les biens qu'il nous donne

Couronnent les efforts d'un peuple vertueux;

Le Canada prospère

Sous les soins d'un tel Père

Et parmi tant d'Etats aucun n'est plus heureux.

Son Règne en vain du monde a vu changer la face

Albion sous ses lois a su tout surmonter...

Sur son Empire auguste

Le fléau des mortels pourrait-il l'emporter.

[17-] Quand ce tyran ${ }^{11} \mathrm{chez}$ lui voulut porter la

Le Monarque entouré d'un Peuple belliqueux [guerre,

Lui fit voir que tout fils de la brave Angleterre,

Préfère à l'Esclavage un trépas glorieux...

11 Napoléon. 
Trop fortuné brigand en vain dans ton délire

Tu crois du juste sort éviter les revers,

Notre Roi, de son isle ébranle ton Empire

En donnant des vertus l'exemple à l'univers, Albion triomphante

Dieu dans ta main puissante

Pour punir les méchants mit le sceptre des mers.

(16-17)

Dans un discours émaillé de rappels de l'Antiquité - "Jeux Olympiques", "Héros de la célèbre Grèce", "Mars", "Jeux Pythiens", "le Parnasse" (17-18), - François Romain félicite alors les gagnants. Un dénommé Mitchel, qui reçoit la médaille au nom de Fleming, fait l'éloge de la Société: elle propage en effet des idées si louables sur l'heureuse constitution britannique! Aussi, "such instruction from a young Society to a number of respectable hearers who are present on this day, must have proved highly satisfactory, and shews... the laudable pursuits of the Society ..." (19). La brochure se clôt sur une autre ode pétrie des mêmes sentiments que les précédents, mais avec un peu plus de virilité. De plus, l'auteur répudie les doutes dont on a parfois aspergé la loyauté canadienne.

... Lo! our standard triumphantly waves round

See the Sceptre of Freedom, by Monarchy held; [the world,

While the Lightnings of Gaul inoffensively hurld, By the Bulwarks of Britain are stoutly repel'd; Midst the Ruins of Europe unshaken he stands, Extending to Princes his Fatherly hands,

While Britons with Pride, ever firm at his side, Will triumph or sink with the King of the brave, Who lives but to govern, and governs to save.

... Let the Trumpet of Fame Over Europe proclaim,

That his Britons contend for the rights of Mankind...

[21] Ye Freemen stand true, to the storm no

Be Britons, and bid the usurper defiance, [compliance,

From pole unto pole, bid your thunders to roll, And freedom redeem ere she basely expire... 
And you amongst whom is unravelled my lay, Shall my strains an ungen'rous suspicion disclose?

Accurst be the muse that a doubt could betray, Of a People who fought and defeated his foes, Ye freeborn of Lawrence awake at the name, With Britain we move in the sphere of her fame, Then nobly stand fast, to encounter the blast And contribute to save her ere Freedom expire, Or sacrifice all for your King and your Sire. (20-21)

(à suivre)

JEAN-PIERRE WALLOT

Musée national, Ottawa, Ont.

VAUGEoIs, Denis, La civilisation française et catholique au Canada - Cours général et cours scientifique - 11e année. Guide à l'intention des maîtres pour l'année 1966/67. Edition revue et complétée. Ministère de l'éducation. Direction générale des Programmes et des Examens, 1966.

Nous devons ce programme de l'enseignement de l'Histoire du Canada à $M$. Denis Vaugeois et à quelques-uns de ses collaborateurs. C'est plus qu'un schéma présenté sèchement. L'enseignant y peut trouver quelques amples développements, tantôt de la main des auteurs, tantôt empruntés aux historiens. On y peut noter un excellent esprit. Le titre seul dit déjà quelque chose. Les auteurs ne prétendent infléchir l'histoire ni du côté de l'optimisme excessif, ni du côté du pessimisme desséchant. Le choix des historiens appelés à fournir de plus larges renseignements procède d'une parfaite probité. Il $\mathrm{y}$ a donc, en ce programme, les éléments d'un excellent manuel d'Histoire canadienne-française. Il n'y a plus qu'à former le souhait que ce manuel prenne forme le plus tôt possible.

LIONEL GroulX, ptre 